\title{
HIGH-ENERGY NEUTRINOS FROM GALACTIC SOURCES
}

\author{
Alexander Kappes ${ }^{a}$ \\ Friedrich-Alexander-Universität Erlangen-Nürnberg, Erlangen Centre for \\ Astroparticle Physics, Erwin-Rommel-Str. 1, 91058 Erlangen, Germany \\ Abstract. Even 100 years after the discovery of cosmic rays their origin remains a \\ mystery. In recent years, $\mathrm{TeV}$ gamma-ray detectors have discovered and investi- \\ gated many Galactic sources where particles are accelerated up to energies of 100 \\ $\mathrm{TeV}$. However, it has not been possible up to now to identify these sites unambigu- \\ ously as sources of hadronic acceleration. The observation of cosmic high-energy \\ neutrinos from these or other sources will be a smoking-gun evidence for the sites \\ of the acceleration of cosmic rays.
}

\section{Introduction}

Up to now, information on objects in our galaxy and beyond has nearly exclusively been obtained using electromagnetic waves as cosmic messengers. Modern astronomy started in 1610 with optical photons, when Galileo Galilei took one of the first telescopes and pointed into the sky. Only in the last century, people started to extended the observations to lower and higher energies of the electromagnetic spectrum, where today's instruments span an enormous range of 20 orders of magnitude from radio waves to $\mathrm{TeV}$ gamma-rays.

In addition, we know from measurements of the cosmic-ray spectrum that there exist sources in the universe which accelerate protons or heavier nuclei up to energies of $\sim 10^{20} \mathrm{eV}, 10^{7}$ times higher than the most energetic manmade accelerator, the LHC at CERN. These highest energies are believed to be reached in extra-Galactic sources like gamma-ray bursts or active galactic nuclei whereas Galactic sources like supernova remnants (SNRs) or micro-quasars are thought to accelerate particles at least up to energies of $3 \times 10^{15} \mathrm{eV}$, also called the "knee" region of the cosmic-ray spectrum. Though these phenomena are quite different, the basic acceleration mechanism is believed to be very similar. Particles are injected into shock fronts which develop when fast moving matter collides with other matter. In these shock fronts, the injected particles are accelerated in a repeating process (Fermi acceleration) where the energy gain per cycle is only small. Other phenomena where a "one-shot" acceleration of cosmic rays to high energies might take place are objects with strong magnetic fields (up to $10^{15} \mathrm{G}$ ) like pulsars and magnetars. However, despite the detailed measurements of the cosmic-ray spectrum and 100 years after their discovery by Victor Hess, we still do not know what the sources of the cosmic rays are as they are deflected in the Galactic and extra-Galactic magnetic fields and hence have lost all information about their origin when reaching Earth. Only at the highest energies beyond $\sim 10^{19.6} \mathrm{GeV}$ cosmic rays might retain enough directional information to locate their sources.

\footnotetext{
${ }^{a}$ e-mail: kappes@physik.uni-erlangen.de
} 
Alternative messengers for locating the sources of the cosmic rays must have two distinct properties: they have to be electrically neutral and essentially stable. Only two of the known elementary particles meet these requirements: photons and neutrinos. Both particles are inevitably produced when the accelerated protons or nuclei collide with matter or photons inside or near the source. In these reactions neutral and charged pions are produced which then decay into high-energy photons and neutrinos, respectively. The well known ratio between the production of neutral and charged pions yields a direct link between the photon and neutrino flux from proton-proton and proton-gamma interactions, respectively.

The number of sources detected in $\mathrm{TeV}$ gamma-rays has increased dramatically during the last decade which was made possible by significant advances in the technique of air Cherenkov telescopes. Today, over 100 sources are known, both Galactic (e.g., SNRs, micro-quasars, pulsars) and extra-Galactic (e.g., active galactic nuclei, starburst galaxies). However, it has not been possible up to now to unambiguously identify these sources as sites of cosmic-ray acceleration as the observed $\mathrm{TeV}$ photons could also have been generated in the upscattering of photons in reactions with accelerated electrons (inverse-Compton scattering). High-energy neutrinos, on the other hand, are only produced in reactions of accelerated hadrons and hence are a smoking-gun evidence for the sources of cosmic rays.

\section{Potential Galactic neutrino sources}

From the discussion above it follows naturally that $\mathrm{TeV}$ gamma-ray sources are prime candidates for high-energy neutrino emission. Today, the list of Galactic objects with observed TeV gamma-ray emission contains a.o. SNRs, pulsar wind nebulae (PWNe), binary systems and molecular clouds. In the following, we will discuss the prospects for neutrino emission from these objects and the expected fluxes. For an overview and more details about the sources see [1] and references therein.

Supernova remnants: SNRs are the prime candidate for the acceleration of the Galactic cosmic rays. This has several reasons: There exists a plausible model for the acceleration of hadrons in the shock fronts generated when the ejecta of the supernova interact with the surrounding medium. Furthermore, energetic considerations show 2] that the observed supernova rate of about 3 per century and a conversion of $10 \%$ of the $10^{51} \mathrm{erg}$ released per nova into cosmic-ray acceleration matches quite well the energy required to keep the energy in cosmic rays constant over time. Up to now, 11 SNRs have been observed in $\mathrm{TeV}$ gamma-rays. Under the assumption that all gamma-ray photons origin from the interaction of accelerated hadrons and utilizing the connection between gamma-rays and neutrinos one can make rather precise predictions 

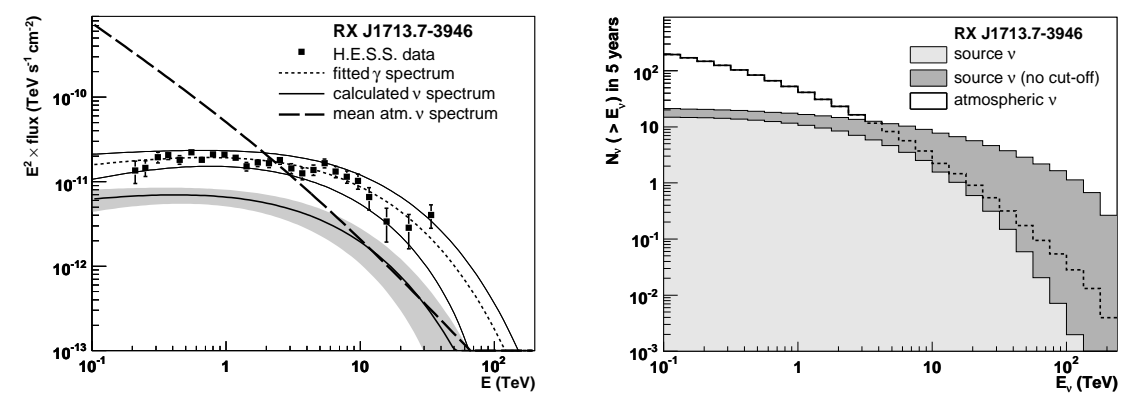

Figure 1: Left: Measured photon and expected neutrino flux from RX J1713.7-3946. The area formed by the solid lines and the shaded area represent the uncertainty (including systematics) on the respective spectrum. Right: Expected event rates from RX J1713.7-3946 in a cubic-kilometer sized neutrino telescope with and without a high-energy cut-off. Also shown is the rate of atmospheric neutrinos. Taken from [3].

for the expected neutrino flux from these sources [3]. An example of such a calculation is shown in the left plot of Fig. 1. Using the effective muon neutrino area of a neutrino telescope one can calculate the integrated number of observed events as a function of the threshold energy. This is displayed in the right plot of Fig.1 for a water Cherenkov neutrino telescope with an instrumented volume of $1 \mathrm{~km}^{3}$. The importance of the cut-off at high energies for the detectability of the source becomes apparent when comparing the light and dark shaded areas with the expected background from atmospheric neutrinos. The strong contribution of high-energy neutrinos to the number of detected events origins from the strong rise of the effective area with the neutrino energy which is mainly caused by the increasing neutrino-nucleon cross section and the extended range of the muons produced. On the other hand, the number of background events scales with the size of the emission region which in the case of RX J1713.7-3946 is quite large with $1.3^{\circ}$ diameter. These two features, the low energy cut-off and the large emission region, make the detection of this and similar sources like RX J0852.0-4622 with neutrino telescopes challenging. In addition, there exits a population of SNRs where no cut-off could be determined from the measured gamma-ray spectrum due to the large error bars at high energies. However, these spectra are rather steep and only a small number of events comparable to those from atmospheric neutrinos is expected [3].

Binary systems: The binary systems observed in gamma-rays consist of a compact object (either a neutron star or a black hole) and a companion star. The compact object accretes material from the companion star producing jets where particles are accelerated. One of these objects is LS 5039 discovered with the H.E.S.S. telescope. The system has a period of 3.9 days and the $\mathrm{TeV}$ 
gamma-ray emission region is point-like for neutrino telescopes. A calculation of the expected neutrino event rate from the measured gamma-ray flux yields less than one event per year for a cubic-kilometer neutrino telescope [3]. However, due to the dense photon fields originating from the companion star the measured gamma-ray flux may be significantly suppressed by up to a factor 100 [4. If this is indeed true then this and similar sources would be one of the most promising targets for neutrino telescopes.

Pulsar wind nebulae: A PWN is powered by the wind from a pulsar which streams into the ambient medium creating shock fronts. In these shock fronts particles are accelerated. In general, PWNe are believed to accelerate mainly electrons. In [5] however, the authors argue that there also might be a significant fraction of nuclei in the pulsar wind. In this case PWNe like Vela X pose interesting targets for neutrino telescopes.

Molecular clouds: Gamma-ray emission from molecular clouds origins from the interaction of cosmic rays from a nearby source, e.g., a SNR, with the nuclei in the cloud. Hence, molecular clouds are a "guaranteed" source of neutrino emission. Such an emission region was located by H.E.S.S. near the Galactic center. Unfortunately, the expected neutrino fluxes derived from the measured gamma rays are rather low and the emission region is quite large making the detection of this and other molecular clouds in neutrinos very challenging.

\section{The missing Pevatrons}

The existence of the "knee" in the cosmic ray spectrum tells us that there must exist Galactic cosmic-ray sources which accelerate protons up to energies of several PeV. These "Pevatrons" will produce pionic gamma rays whose spectrum extends to several hundred $\mathrm{TeV}$ without cut-off in interactions with the interstellar medium. However, none of the observed gamma-ray sources shows such a spectrum. One reason for this observation could be the fact that the highest energies in SNRs are only reached during the first few hundred years after the supernova explosion (a SNR has a typical lifetime of the order of 10,000 years). In this case, it could simply be possible that currently there does not exist an observable SNR in this early phase. Nevertheless, the detection of these cosmic rays could still be possible by observing the gamma rays produced in their interaction with interstellar medium, in particular, with dense molecular clouds as shown in [6. In [7] the authors argue that some of the gamma-ray sources discovered by Milagro 8 might origin from such secondary interactions of cosmic rays from Pevatrons. Future measurements with air Cherenkov and neutrino telescopes have to show whether this is true. On the other hand, one of the suggested sources, MGRO 1908+06, has now been plausibly associated with a pulsar [9], disfavoring it as a region of proton acceleration. 


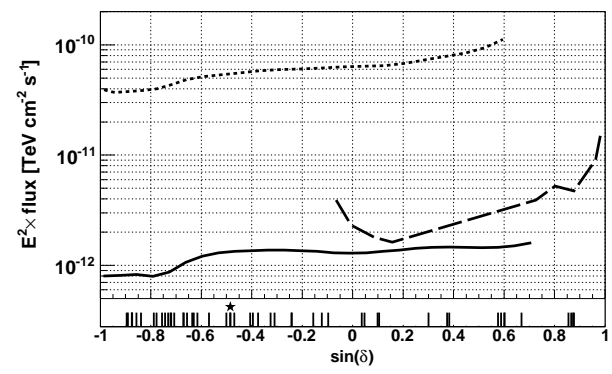

Figure 2: Upper part: Sensitivity at 90\% CL of neutrino telescopes to an $E^{-2}$ neutrino flux as a function of the source declination: ANTARES (dotted, predicted) [10], IceCube (dashed, predicted) [12, KM3NeT (solid, not final), [13. Lower part: declination of Galactic objects with observed $\mathrm{TeV}$ gamma-ray emission. The position of the Galactic Center is marked with a star.

\section{Sensitivity of current and future neutrino telescopes}

All current event rate calculations indicate that neutrino telescopes with instrumented volumes of cubic kilometer size are necessary to identify the first cosmic source of high-energy neutrino emission. Due to the large background of downgoing muons produced in interactions of cosmic rays with the Earth's atmosphere, neutrino telescopes have their highest sensitivity when looking through the Earth. Hence, in order to cover the full sky with high sensitivity both a telescope in the Northern and in the Southern hemisphere is necessary.

Currently, the most sensitive neutrino telescope in the Northern hemisphere is the ANTARES detector [10, installed at a depth of $2500 \mathrm{~m}$ off the coast of south France in the Mediterranean Sea. The detector instruments a volume of about $0.01 \mathrm{~km}^{3}$ and therefore is probably too small for cosmic neutrino detection. In the Southern hemisphere, the currently largest and hence most sensitive neutrino telescope, IceCube, is close to completion planned for next year. With an instrumented volume of $1 \mathrm{~km}^{3}$ it is the first cubic-kilometerclass detector. The sensitivities of both experiments to an $E^{-2}$ neutrino flux are shown in Fig. 2. It is apparent, that in order to achieve a high-sensitivity coverage over the full sky a cubic kilometer class detector in the Northern hemisphere is needed which will cover most of the Galactic plane including the Galactic center. Such a detector with an instrumented volume of about $5 \mathrm{~km}^{3}$, KM3NeT [11, is currently in its planning phase. Data taking is planned to start around 2014.

\section{$5 \quad$ Summary and outlook}

The observation of cosmic high-energy neutrinos will open a new window to the universe and help to solve long-standing mysteries like the question of the origin 
of the cosmic rays. With the recent advances in gamma-ray astronomy we were able to identify many good Galactic candidate sources of neutrino emission. However, due to the expected low fluxes and the early high-energy cut-offs of the predicted neutrino spectra the detection of these sources is challenging.

IceCube will be the first detector to advance into the "discovery region" but will likely only scratch it. Therefore, a factor five to ten more sensitive detector is needed in the Northern hemisphere which will also cover the inner Galactic Plane and Center. This detector, KM3NeT, is supposed to start data taking around 2014. With IceCube and KM3NeT combined, neutrino astronomy will hopefully soon become reality.

\section{Acknowledgments}

Supported by the BMBF under project 05A08WEA. The author also acknowledges the support by the EU Marie Curie OIF program.

\section{References}

[1] F. Aharonian, J. Buckley, T. Kifune, and G. Sinnis. Rep. Prog. Phys., 71:096901, 2008.

[2] F. Halzen. J. Phys.: Conf. Series, 171:012014, 2009.

[3] A. Kappes, J. Hinton, C. Stegmann, and F. A. Aharonian. ApJ, 656:870, 2007.

[4] F. A. Aharonian, L. A. Anchordoqui, D. Khangulyan, and T. Montaruli. J. Phys.: Conf. Series, 39:408, 2006.

[5] D. Horns, F. Aharonian, A. Santangelo, A. I. D. Hoffmann, and C. Masterson. A\&A, 451:L51, 2006.

[6] S. Gabici and F. A. Aharonian. ApJ, 665:L131, 2007.

[7] F. Halzen, A. Kappes, and A. Ó Murchadha. Phys. Rev., D78:063004, 2008.

[8] A. A. Abdo, (Milagro Collaboration), et al. ApJ, 664:L91, 2007.

[9] A. A. Abdo, (Fermi-LAT Collaboration), et al. ApJ, 711:64, 2010.

[10] P. Coyle, (ANTARES Collaboration), et al., Preprint arXiv:1002.0754, 2010.

[11] P. Bagley, (KM3NeT Consortium), et al. KM3NeT, conceptual design for a deep-sea research infrastructure incorporating a very large volume neutrino telscope in the Mediterranean Sea, 2008. ISBN 978-90-6488031-5.

[12] J. Dumm, (IceCube Collaboration), et al. All-sky point-source search with 40 strings of IceCube. to be published in Proc. of ICRC'09.

[13] U. F. Katz, (KM3NeT Consortium), et al. The KM3NeT project. to be published in Proc. of the VLVnT'09 Workshop. 